\title{
A comparison of bread wheat genotypes in various environments using different stability models
}

Said, A.A. *, M.H. Motawea, and H. Morsy

\author{
Department of Agronomy, Faculty of Agriculture, Sohag University, 82524 Sohag, Egypt
}

\begin{abstract}
Twenty-five bread wheat genotypes were tested under eight environments ( 2 growing seasons $\times 4$ water stresses) to study stability and adaptability for physiological and agronomic traits. Analysis of variance showed highly significant variations among all tested genotypes for all studied traits; chlorophyll content, canopy temperature, specific leaf area, spike length, no. of spike/plant, 1000kernel weight and grain yield. The results indicated that Line 9 (13.21 ardab/feddan) beside Sids 1 (13.19 ardab/feddan) and Misr 2 (11.91 ardab/feddan) cultivars were observed as most stable and widely adapted across environments, surprised mean performance for grain yield than grand mean, regression coefficient $\left(b_{i}{ }^{\text {Eberhart }}\right.$ and $\left.b_{i}{ }^{\text {Freeman }}\right)$ equal or near to one, $\mathrm{B}_{i}{ }^{\text {Perkins }}$ equal or near to zero and mean square deviation from regression $\left(S^{2} d_{i}^{\text {Eberhart }}\right.$ and $\left.S^{2} d_{i}^{\text {Freeman }}\right)$ non-significant from zero, according to previous models. Moreover, Line 9 showed also stability for number of spikes/plant, 1000 kernel weight and chlorophyll concentration under various environments; it was considered to be superior for grain yield across different environments. Meanwhile, the highest yielding genotype (Line 20) showed stability only under favorable conditions (surpassed the grand mean by 15.58 ardab/feddan, $b_{i}{ }^{E}$ and $b_{i}{ }^{\mathrm{F}}>1, B_{i}>0$ and $S^{2} d_{i}{ }^{E}$ and $S^{2} d_{i}{ }^{F}$ non-significant), it could be promoted to breeding program with a stable genotype (Line 9) over different environments to obtain a stable variety across environments with a high yield.
\end{abstract}

Keywords: Stability models; Performance; Environments; Triticum aestivum.

\section{Introduction}

Wheat (Triticum aestivum L.) is important most strategic crop in in the world as well as Egypt.It is a daily source of carbohydrates of the majority of Egyptian citizen. Wheat area harvested in Egypt was 1.3 million ha produced 8.80 million metric tons with an average of $6689.5 \mathrm{~kg} / \mathrm{ha}$ (FAO STAT, 2018) while, wheat consumption in 2017-2018 estimate of 19.8 million tones (USDA STAT, 2018). There is a big difference between production and consumption of Egyptian

\footnotetext{
*Corresponding author: alaa.said@agr.sohag.edu.eg Received: October 23, 2020;

Accepted: November 18, 2020;

Published: November 24, 2020.
}

wheat. To reduce the gap between production and consumption, we need to expand growing wheat in new environments due to the limited area of the agriculture land, but these new environments suffer from some abiotic stresses, such as heat, drought stress and salinity.

Drought or water deficit is one of the environmental stresses that severely influence the wheat growth, development, and production (Werteker et al., 2010; Ongom et al., 2016). The response of wheat plants to water deficit stress is a function of genotypes, intensity and duration of stress, weather conditions and stages of crop growth and development. It should be noted that stress 
occurrence time is more important than drought stress intensity. Grain yield stability is one of the most important goals of breeding programs, especially in subtropical environments. Some physiological traits have the potential to improve crop performance under abiotic stress (Chaves et al. 2003; Condonet al. 2004; Richards 2006).Better understanding of the genetic basis of physiological trait variability will improve the efficiency of wheat for drought tolerance. The features of stable genotype are complex due to genotype $\mathrm{x}$ environment interactions (GEI) (Alwala 2010; Moghaddam et al. 2013). Hence a study of GEI can lead to successful evaluation of wheat cultivars for stability in yield performance under various environmental conditions. Almost all breeders have used the term "stability" to characterize a genotype which ever showed a constant yield across environments (Dehghani et al. 2008; Changizi et al. 2014).Several statistical methods can be used as important measures of crop yield stability, the most widely used is the joint linear regression analysis as proposed by (Eberhart and Russell, 1966; Perkins and Jink, 1968; Freeman and Perkins, 1971).The selection of stable genotypes, based on stability parameters, caused high yield genotypes to be introduced as stable genotypes. The aims of thisstudy were to estimate stability parameters of the 25 bread wheat genotypes under 8 environments (two year and four water irrigation treatments) to identify genotypes with high yield stability.

\section{Materials and Methods}

Twenty five bread wheat (Triticum aestivum L.) genotypes from diverse origin including 5 local varieties and 20 inbred lines $\left(\mathrm{F}_{8}\right)$ were used in this study (Table 1). Twofield experiments were conducted at the experimental farm of Faculty of Agriculture, Sohag University, Sohag, Egypt. The field experiments were created in eight different environments, 2 years (2017/2018 and $2018 / 2019$ growing seasons) and four water irrigation treatments; normal irrigation (10 irrigation frequencies), skipping two irrigation at the tillering stage, skipping two irrigation at the booting stage and skipping two irrigation at the milk-ripe stage.

The experimental design was a randomized complete block with a split-plot arrangement of treatments, with three replications. The irrigation treatments were allocated to the main plots and the 25 genotypes were randomly distributed to the sub plots. Each genotype was sown in two rows $3.0 \mathrm{~m}$ long and $30 \mathrm{~cm}$ between rows and $10 \mathrm{~cm}$ within rows (sub plot size $=2 \times 0.30 \times 3.0=1.8 \mathrm{~m}^{2}$ ). The experimental field soil was sandy clay in texture. All recommended cultural practices were applied.

Data were recorded on specific leaf area (leaf area in $\mathrm{cm}^{2}$ produced $\mathrm{g}^{-1}$ leaf dry weight plant $\left.^{-1}\right)$, chlorophyll concentration $\left(\mathrm{mg} \mathrm{cm}^{-2}\right)$ was determined as chlorophyll index using Chlorophyll Meter (SPAD-502), canopy temperature $\left({ }^{\circ} \mathrm{C}\right)$ using infrared thermometer (assessment of all physiological traits were performed after anthesis stage on flag leaf area in plants), no. of spikes/plant, spike length (cm), 1000-kernel weight (gm) and grain yield (ard./fed.): It was determined from the whole grain yield of each sub plot in terms of $\mathrm{kg} / \mathrm{plot}$ and converted to ardab ( $\operatorname{ardab}=150 \mathrm{~kg}$ ) per feddan (feddan $=0.42$ hectare).

The combined analysis of the variance was performed according to Gomez and Gomez (1994). Means were compared by Revised Least Significant Difference (R. LSD) at 5\% level of significant (Steel \&Torrie, 1981). A correlation among stability parameters was performed by using simple correlation (Fisher $\&$ Yates, 1953). The stability analysis was computed as obtained by Eberhart and Russell 
model (1966), Perkins and Jinks model (1968)

and Freeman and Perkins model (1971).

Table 1. Pedigree description of 25 bread wheat genotypes evaluated in eight environments during 2017/2018 and 2018/2019 growing season.

\begin{tabular}{|l|l|}
\hline Genotype & \multicolumn{1}{|c|}{ Pedigree } \\
\hline Line 1 to 10 & $\begin{array}{l}\text { Derived from a cross between Sids 4 x Tokwie; } \\
\text { Sids 4: High yielding local variety, while Tokwie: Drought tolerant variety } \\
\text { introduced from South Africa. }\end{array}$ \\
\hline Line 11 to 20 & $\begin{array}{l}\text { Derived from a cross between Sids 4 x Kasyon/glennson-81; } \\
\text { Kasyon/glennson-81: Drought tolerant variety Introduced from ICARDA. }\end{array}$ \\
\hline Shandweel 1 & $\begin{array}{l}\text { Site / Mo /4/ Nac / Th.Ac // 3* Pvn /3/ Mirlo / Buc CMSS93B00567S-72Y- } \\
\text { 010M-010Y-010M-3Y-0M-0THY- 0SH. }\end{array}$ \\
\hline Sids 1 & HD2172/Pavon"s"//1158.57/Maya74"s" \\
\hline Sids 12 & $\begin{array}{l}\text { BUC//7C/ALD/5/MAYA74/ON//1160.147/3/BB/GLL/4/C } \\
\text { HAT"S"/6/MAYA/VUL//CMH74A.630/4*SXSD7096-4SD-1SD-1SD-0SD. }\end{array}$ \\
\hline Giza 168 & MIL/BUC//Seri CM93046-8M-0Y-0M-2Y-0B. \\
\hline Misr 2 & SKAUZ/BAV92.CMSS96M03611S-1M-010SY-010M-010SY-8M-0Y-0EGY. \\
\hline
\end{tabular}

\section{Results and discussion}

\section{1- Analysis of variance:}

Combined analysis of variance of the studied traits (Table 2) revealed that all the variations in the total sum of squares were attributed to the various environmental factors ( $\mathrm{Y}$ and $\mathrm{D}$ ) and their interactions which always were statistically highly significant with the exception of year effect of chlorophyll content and $Y \times D$ interaction of specific leaf area, spike length, no. of spikes/plant and grain yield. The analyzed data also showed that there were highly significant differences among genotypes for all the studied plant characters across environments. Obviously, all degrees of $\mathrm{G} \times \mathrm{E}$ interactions were significant with exception of $Y \times G$ of 1000 -kernel weight and $\mathrm{Y} \times \mathrm{D} \times \mathrm{G}$ of chlorophyll content and 1000kernel weight (Table 2). The genetic diversity and the significant $\mathrm{G} \times \mathrm{E}$ interactions exhibited both sensitivity of genotypes and differential responses of these genotypes to variable environments, suggesting the importance of stability parameters assessment of these genotypes under these conditions to identify the best stable suitable genotypes under this range of environments. Similar results were obtained by (EI-Morshidy et al., 2000; Kheiralla et al., 2004; Bose et. al., 2014; Mohamed and Said 2014 and Ibrahim and Said 2020).

\section{2- Mean performance and stability models:}

Mean performance and the estimates of stability parameters using three models viz., Eberhart and Russell's, Perkins and Jinks' and Freeman and Perkins' obtained for agronomic and physiological data are presented in Tables (4, 5, 6 and 7).

\section{3- Number of spikes/plant:}

The mean number of spikes/plant ranged from 4.94 (Line 19) to 7.46 spikes/plant (Line 7), the differences being significant with an average of 6.23 spikes/plant (Table 4). According to Eberhart and Russell's and Perkins and Jinks' models (Table 4 and Fig. 1), the genotypes Line 9, Shandweel 1 and Misr 2 were stable in varied environmental conditions (highest number of spikes/plant compared with grand mean overall genotypes, 
Table 2: Mean squares of the combined analysis of variance for all studied traits.

\begin{tabular}{|l|c|c|c|c|c|c|c|c|}
\hline \multirow{2}{*}{ S.o.v } & \multirow{2}{*}{ df } & \multicolumn{7}{|c|}{ Mean Squares } \\
\cline { 3 - 9 } & & $\begin{array}{c}\text { Chlorophyll } \\
\text { content }\end{array}$ & $\begin{array}{c}\text { Canopy } \\
\text { temp. }\end{array}$ & $\begin{array}{c}\text { Specific } \\
\text { leaf area }\end{array}$ & $\begin{array}{c}\text { Spike } \\
\text { length }\end{array}$ & $\begin{array}{c}\text { No. of } \\
\text { spike } \\
\text { /plant }\end{array}$ & $\begin{array}{c}1000- \\
\text { kernel } \\
\text { weight }\end{array}$ & $\begin{array}{c}\text { Grain yield } \\
\text { Years (Y) }\end{array}$ \\
\cline { 3 - 10 } & 1 & 9.79 & $298.77^{* *}$ & $2476.71^{* *}$ & $0.144^{* *}$ & $1.10^{* *}$ & $80.73^{* *}$ & $3391.72^{* *}$ \\
\hline Error a & 2 & 5.25 & 5.89 & 2039.7 & 0.018 & 0.281 & 9.93 & 57.96 \\
\hline Drought (D) & 3 & $883.03^{* *}$ & $952.08^{* *}$ & $167097.7^{* *}$ & $158.63^{* *}$ & $121.71^{* *}$ & $611.3^{* *}$ & $145351.3^{* *}$ \\
\hline Y x D & 3 & $24.82^{* *}$ & $534.3^{* *}$ & 887.11 & 0.059 & 0.03 & $24.51^{* *}$ & 37.1 \\
\hline Error b & 12 & 2.01 & 2.96 & 1148.39 & 0.064 & 0.122 & 7.77 & 120.42 \\
\hline Genotypes (G) & 24 & $233.19^{* *}$ & $565.73^{* *}$ & $2664.29^{* *}$ & $21.8^{* *}$ & $9.85^{* *}$ & $6.6^{* *}$ & $6640.74^{* *}$ \\
\hline Y x G & 24 & $0.738^{* *}$ & $550.6^{* *}$ & $1605.88^{* *}$ & $0.127^{* *}$ & $0.15^{* *}$ & 1.45 & $340.02^{* *}$ \\
\hline D x G & 72 & $28.2^{* *}$ & $540.71^{* *}$ & $2949.96^{* *}$ & $1.73^{* *}$ & $1.164^{* *}$ & $3.73^{*}$ & $1738.81^{* *}$ \\
\hline Y x D x G & 72 & 0.968 & $515.15^{* *}$ & $1246.68^{* *}$ & $0.083^{*}$ & $0.133^{* *}$ & 1.41 & $154.23^{* *}$ \\
\hline Pooled error & 348 & 3.42 & 6.70 & 205.14 & 0.056 & 0.065 & 2.53 & 42.4 \\
\hline
\end{tabular}

*,**Significant at 5 and $1 \%$ levels of probability, respectively.

regression coefficient $\left(\mathrm{b}_{\mathrm{i}}^{\mathrm{E}}\right)$ equal or near to unity, Bi equal or near to zero and deviations from regression coefficient $\left(\mathrm{S}^{2} \mathrm{di}^{\mathrm{E}}\right)$ closed zero or non-significant). Mohamed and Said, 2014 showed that eight wheat genotypes were acceptable production statistics of both responses and stability for the number of spikes/plant (high average comparing to the grand mean, and insignificant bi and $\mathrm{S}^{2} \mathrm{di}$ ). Similar results were reported by EI-Morshidy et al. (2000), Kheiralla et al. (2004) and Ibrahim and said (2020). While lines 1, 5, 8, 13 and 19 were stable (bi ${ }^{\mathrm{E}}$ equal or near unity, Bi equal or near zero and $\mathrm{S}^{2} \mathrm{di}^{\mathrm{E}}$ non-significant) but had low number of spikes/plant. On the other hand, according to Freeman and Perkins' model genotypes Line 4, Line 9, Line 17, Shandweel 1 and Misr 2 exhibited highest number of spikes/plant across various environments, bi ${ }^{\mathrm{F}}$ equal or near unity and $\mathrm{S}^{2} \mathrm{di}^{\mathrm{F}}$ non-significant. While, Lines 5, 6, 8, 13 and 19 were stable but gave lower than overall mean number of spikes/plant. The results in this study are generally in harmony with (Saha, 1999 and Islam et al., 2006).

\section{4- Spike length:}

In table 4 , the mean spike length was ranged from $6.40 \mathrm{~cm}$ for Line 12 to $9.90 \mathrm{~cm}$ for Line 20 across environments with an average 7.87 $\mathrm{cm}$. Considering high mean performance than grand mean with stability parameters $\left(b_{i}{ }^{\mathrm{E}}\right.$ and $b_{i}{ }^{F}$ equal or near to one, $B_{i}$ equal or near to zero and $S^{2} d_{i}{ }^{E}$ and $S^{2} d_{i}{ }^{F}$ non-significant), four genotypes namely Line 1, Line 3, Line 15 and Shandweel 1 (Table -3 and Fig. 2) were found desirable and exhibited stable performance across environments for spike length. Meanwhile, fife genotypes namely Lines 5, 17, 18 and Misr 2 were stable in varied environmental conditions but had low spike length when compared with grand mean. Khan et al., 2017 showed that the genotypes CT09141, SRN-09048 and SRN-09063 are highly stable for spike length under different environments, on the bases of bi values $\left(b_{i}=1\right)$ and $\boldsymbol{S}^{2} \boldsymbol{d i v a l u e s ~ n e a r l y ~ e q u a l ~ t o ~ z e r o ~}\left(\boldsymbol{S}^{2} \boldsymbol{d}_{i}=0\right)$.

\section{5- 1000 kernel weight:}

The average of overall tested genotypes for 1000 kernel weight (Table 4) ranged from $31.63 \mathrm{gm}$ for Line 5 to $39.90 \mathrm{gm}$ for Line 9 with an average $37.54 \mathrm{gm}$. Nine genotypes 
Table 3: Estimates of stability parameters based on eight environments using various models for number of spikes/plant and spike length in wheat.

\begin{tabular}{|c|c|c|c|c|c|c|c|c|c|c|c|c|}
\hline Traits & \multicolumn{6}{|c|}{ Number of spikes/plant } & \multicolumn{6}{|c|}{ Spike length $(\mathrm{cm})$} \\
\hline Genotypes & Mean & $\mathrm{b}_{\mathrm{i}}^{\mathrm{E}}$ & $\beta_{\mathrm{i}}$ & $b_{i}^{F}$ & $\begin{array}{c}\mathrm{S}^{2} \mathrm{~d}_{\mathrm{i}} \\
(\mathrm{E})\end{array}$ & $\begin{array}{l}S^{2} d_{i} \\
(F)\end{array}$ & Mean & $\mathrm{b}_{\mathrm{i}}^{\mathrm{E}}$ & $\beta_{\mathrm{i}}$ & $b_{i}{ }^{F}$ & $\begin{array}{l}S^{2} d_{i} \\
(E)\end{array}$ & $\begin{array}{l}S^{2} d_{i} \\
(F)\end{array}$ \\
\hline Line 1 & 6.03 & 0.82 & 0.18 & 0.79 & 0.04 & 0.24 & 9.86 & 1.11 & -0.11 & 1.00 & 0.01 & 0.65 \\
\hline Line 2 & 5.50 & 0.85 & 0.15 & 0.84 & $0.20^{* *}$ & 1.54 & 8.25 & 1.06 & -0.06 & 1.00 & $0.47^{* *}$ & $1.90^{* *}$ \\
\hline Line 3 & 5.30 & 0.80 & 0.20 & 0.77 & $0.35^{\star \star}$ & 2.02 & 8.08 & 1.17 & -0.17 & 1.16 & 0.09 & 0.63 \\
\hline Line 4 & 6.79 & 1.08 & -0.08 & 1.15 & $0.48^{\star *}$ & 2.66 & 8.10 & 1.30 & -0.30 & 1.20 & 0.10 & 0.73 \\
\hline Line 5 & 5.85 & 0.90 & 0.10 & 0.87 & 0.05 & 0.88 & 6.63 & 1.03 & -0.03 & 0.92 & 0.27 & 1.65 \\
\hline Line 6 & 5.58 & $1.25^{\star *}$ & -0.25 & 1.18 & 0.00 & 2.53 & 7.50 & 0.77 & 0.23 & 0.78 & 0.18 & 1.11 \\
\hline Line 7 & 7.46 & $0.68^{* *}$ & 0.32 & $0.67^{\star \star}$ & 0.10 & 0.04 & 7.09 & 0.42 & 0.58 & $0.38^{* *}$ & 0.04 & $0.61-$ \\
\hline Line 8 & 5.78 & 1.06 & -0.06 & 1.10 & 0.01 & 2.07 & 7.77 & 1.19 & -0.19 & 1.08 & $0.34^{*}$ & $2.05^{\star *}$ \\
\hline Line 9 & 6.30 & 1.05 & -0.05 & 1.12 & 0.09 & 2.15 & 6.50 & 0.84 & 0.16 & 0.81 & 0.07 & 0.59 \\
\hline Line 10 & 6.83 & $1.49^{*}$ & -0.49 & 1.36 & $0.56^{\star *}$ & $6.26^{\star *}$ & 9.66 & 1.43 & -0.43 & 1.35 & 0.13 & 0.66 \\
\hline Line 11 & 6.85 & $0.73^{* *}$ & 0.27 & 0.77 & -0.01 & 0.25 & 8.14 & 0.68 & 0.32 & 0.64 & 0.11 & 0.67 \\
\hline Line 12 & 6.63 & 0.69 & 0.31 & 0.59 & 0.31 & 1.02 & 6.40 & 0.48 & 0.52 & $0.43^{* *}$ & -0.04 & -0.27 \\
\hline Line 13 & 5.57 & 0.95 & 0.05 & 0.86 & 0.01 & 0.85 & 7.53 & 1.59 & -0.59 & 1.58 & $1.68^{* *}$ & $7.35^{\star *}$ \\
\hline Line 14 & 5.42 & $1.46^{\star \star}$ & -0.46 & 1.43 & 0.03 & $4.40^{*}$ & 9.68 & 1.38 & -0.38 & 1.41 & $0.32^{*}$ & $1.32^{* \star}$ \\
\hline Line 15 & 6.99 & $1.97^{\star *}$ & -1.29 & 1.93 & 0.00 & $11.29^{\star *}$ & 8.15 & 1.18 & -0.18 & 1.13 & 0.00 & 0.33 \\
\hline Line 16 & 6.67 & $1.57^{\text {** }}$ & -0.57 & 1.51 & $0.36^{\star *}$ & $5.95^{\star}$ & 7.82 & 1.16 & -0.16 & 1.16 & $0.73^{* *}$ & $3.26^{* *}$ \\
\hline Line 17 & 7.29 & 0.89 & 0.11 & 0.85 & $0.17^{\star \star}$ & 1.16 & 7.02 & 1.08 & -0.08 & 1.01 & 0.09 & 0.66 \\
\hline Line 18 & 5.58 & $0.42^{\star *}$ & 0.58 & $0.43^{* *}$ & $0.17^{\star *}$ & -0.26 & 7.36 & 1.05 & -0.05 & 0.98 & 0.13 & 0.73 \\
\hline Line 19 & 4.94 & 0.91 & 0.09 & 0.84 & 0.05 & 0.58 & 6.45 & 0.59 & 0.41 & $0.56^{* *}$ & 0.09 & 0.64 \\
\hline Line 20 & 6.09 & $0.58^{* *}$ & 0.42 & $0.51^{*}$ & 0.02 & -0.46 & 9.90 & 1.32 & -0.32 & 1.31 & 0.26 & 1.28 \\
\hline Shandweel 1 & 6.70 & 1.13 & -0.13 & 1.11 & 0.04 & 2.11 & 8.26 & 0.99 & 0.01 & 0.97 & 0.00 & 0.24 \\
\hline Sids 1 & 5.98 & $0.67^{\star *}$ & 0.33 & $0.65^{\text {** }}$ & 0.08 & 0.11 & 7.41 & 0.59 & 0.41 & $0.56^{\star *}$ & -0.02 & 0.29 \\
\hline Sids 12 & 7.34 & 0.80 & 0.20 & 0.79 & $0.15^{\star \star}$ & 0.82 & 8.79 & 0.82 & 0.18 & 0.81 & 0.03 & 0.45 \\
\hline Giza 168 & 5.52 & 1.07 & -0.07 & 0.53 & $-0.20^{\star *}$ & 1.67 & 7.54 & 0.78 & 0.22 & 0.42 & 0.22 & 0.61 \\
\hline Misr 2 & 6.75 & 1.05 & -0.05 & 1.00 & -0.01 & 1.28 & 6.81 & 0.91 & 0.09 & 0.88 & 0.14 & 0.93 \\
\hline Mean & 6.23 & & & & & & 7.87 & & & & & \\
\hline RLSD 5\% & 0.157 & & & & & & 0.146 & & & & & \\
\hline
\end{tabular}

Mean = grain mean yield $($ ardab/feddan $) ; b_{i}{ }^{\mathrm{E}}=$ regression coefficient of Eberhart and Russell; $\beta_{\mathrm{i}}=$ regression coefficient of Perkins and Jinks; $b_{i}{ }^{F}=$ regression coefficient of Freeman and Perkins; $S^{2} d_{i}(E)=$ deviation from regression Eberhart and Russell; $\mathrm{S}^{2} \mathrm{~d}_{\mathrm{i}}(\mathrm{F})=$ residual MS of Freeman and Perkins model.*, ** Significant at the 0.05 and 0.01 probability levels, respectively. 
Table 4: Estimates of stability parameters based on eight environments using various models for1000-kernel weight and Specific leaf area in wheat.

\begin{tabular}{|c|c|c|c|c|c|c|c|c|c|c|c|c|}
\hline Traits & \multicolumn{6}{|c|}{ 1000-kernel weight (g) } & \multicolumn{6}{|c|}{ Specific leaf area $\left(\mathrm{cm} \mathrm{g}^{-1}\right)$} \\
\hline Genotypes & Mean & $\mathrm{b}_{\mathrm{i}}^{\mathrm{E}}$ & $\beta_{\mathrm{i}}$ & $\mathrm{b}_{\mathrm{i}}^{\mathrm{F}}$ & $\mathrm{S}^{2} \mathrm{~d}_{\mathrm{i}}(\mathrm{E})$ & $\mathrm{S}^{2} \mathrm{~d}_{\mathrm{i}}(\mathrm{F})$ & Mean & $\mathrm{b}_{\mathrm{i}}^{\mathrm{E}}$ & $\beta_{\mathrm{i}}$ & $b_{i}{ }^{\mathrm{F}}$ & $\mathrm{S}^{2} \mathrm{~d}_{\mathrm{i}}(\mathrm{E})$ & $\mathrm{S}^{2} \mathrm{~d}_{\mathrm{i}}(\mathrm{F})$ \\
\hline Line 1 & 37.63 & 0.41 & 0.59 & 0.72 & $11.38^{\star *}$ & $21.09^{* *}$ & 239.73 & 1.08 & -0.50 & 1.02 & $890.87^{* *}$ & $8674.86^{* *}$ \\
\hline Line 2 & 37.58 & 1.00 & 0.00 & 1.03 & -0.54 & 3.24 & 221.79 & 1.18 & -0.58 & 1.07 & $430.90^{* *}$ & $6681.47^{* *}$ \\
\hline Line 3 & 39.84 & 1.17 & -0.17 & 1.25 & 0.33 & $5.38^{* *}$ & 222.25 & 1.17 & -0.48 & 0.98 & 252.07 & $4425.98^{* *}$ \\
\hline Line 4 & 34.13 & 0.96 & 0.04 & 0.94 & -0.36 & $6.82^{*}$ & 236.55 & 1.26 & -0.64 & 1.13 & $709.65^{\star *}$ & $6532.99^{* *}$ \\
\hline Line 5 & 31.63 & 1.30 & -0.30 & 1.25 & -0.39 & $1.05^{\star *}$ & 241.11 & $1.59^{* \star}$ & -0.60 & 1.43 & $403.92^{* *}$ & $10077.07^{* *}$ \\
\hline Line 6 & 38.36 & 1.29 & -0.29 & 1.51 & 0.00 & $2.11^{\star *}$ & 244.64 & 0.99 & -0.31 & 0.84 & $571.82^{\star \star}$ & $3464.35^{\star \star}$ \\
\hline Line 7 & 35.04 & 0.94 & 0.06 & 1.02 & 0.55 & $3.26^{*}$ & 230.26 & 1.18 & -0.16 & 0.99 & 194.53 & $4406.39^{\star *}$ \\
\hline Line 8 & 38.56 & 1.10 & -0.10 & 1.28 & -0.46 & $1.99^{*}$ & 215.48 & $0.55^{*}$ & -0.17 & $0.13^{*}$ & $475.41^{*}$ & $1990.98^{\star \star}$ \\
\hline Line 9 & 39.90 & 0.94 & 0.06 & 1.08 & 1.62 & $14.93^{\star *}$ & 225.65 & 1.72 & -0.30 & 1.51 & 4.83 & $9685.94^{\star \star}$ \\
\hline Line 10 & 37.91 & 0.96 & 0.04 & 0.82 & -0.67 & 3.00 & 198.24 & 1.17 & -0.37 & 1.00 & 297.06 & $5465.90^{* *}$ \\
\hline Line 11 & 38.87 & 1.12 & -0.12 & 1.17 & -0.86 & $3.71^{*}$ & 217.82 & $1.47^{\star}$ & -0.10 & 1.38 & $447.36^{\star \star}$ & $9219.66^{\star \star}$ \\
\hline Line 12 & 38.72 & 1.19 & -0.19 & 1.02 & -0.10 & 1.31 & 228.90 & 1.03 & 0.45 & 0.87 & $1041.38^{* *}$ & $3438.68^{* *}$ \\
\hline Line 13 & 38.23 & 0.86 & 0.14 & 0.89 & -0.39 & 1.83 & 222.08 & 0.90 & -0.04 & 0.86 & $1747.20^{\star \star}$ & $11186.45^{\star \star}$ \\
\hline Line 14 & 38.23 & 0.84 & 0.16 & 0.80 & 1.31 & 3.16 & 247.24 & 1.19 & 0.07 & 1.27 & $453.41^{\star *}$ & $8963.20^{* *}$ \\
\hline Line 15 & 38.61 & 0.86 & 0.14 & 0.90 & -0.63 & 2.19 & 242.96 & $1.51^{* *}$ & -0.29 & 1.38 & 244.54 & $8940.67^{\star *}$ \\
\hline Line 16 & 38.42 & 0.74 & 0.26 & 0.87 & 0.77 & 3.27 & 217.52 & $0.70^{*}$ & 0.41 & 0.52 & $386.82^{*}$ & $2994.01^{* *}$ \\
\hline Line 17 & 37.77 & 0.90 & 0.10 & 0.81 & 0.72 & $14.73^{\star \star}$ & 230.45 & $0.31^{* *}$ & 0.61 & $0.23^{*}$ & $465.04^{* *}$ & $1913.50^{* *}$ \\
\hline Line 18 & 34.44 & 0.71 & 0.29 & 0.92 & 1.53 & 4.61 & 227.88 & $1.41^{*}$ & 0.02 & 1.32 & 106.93 & $8040.62^{* *}$ \\
\hline Line 19 & 33.30 & 1.03 & -0.03 & 1.05 & 1.57 & $3.22^{*}$ & 226.59 & $0.19^{* *}$ & 0.08 & $0.08^{* *}$ & $293.33^{*}$ & $1722.72^{\star *}$ \\
\hline Line 20 & 37.61 & 1.35 & -0.35 & 1.22 & 1.28 & $8.60^{\star \star}$ & 219.81 & 0.63 & 0.91 & 0.67 & 61.36 & $2143.00^{* *}$ \\
\hline Shandweel 1 & 38.93 & 1.04 & -0.04 & 1.11 & -0.67 & 2.68 & 226.14 & 0.76 & 0.45 & 0.77 & 299.51 & $3438.897^{* *}$ \\
\hline Sids 1 & 38.53 & 1.05 & -0.05 & 1.02 & -0.30 & $5.57^{\star *}$ & 219.87 & $0.20^{* *}$ & 0.48 & $0.09^{*}$ & $561.86^{\star *}$ & $2490.408^{\star *}$ \\
\hline Sids 12 & 39.29 & 1.20 & -0.20 & 1.24 & -0.46 & $2.48^{* *}$ & 221.01 & 0.84 & 0.44 & 0.84 & 222.93 & $3824.141^{* *}$ \\
\hline Giza 168 & 38.68 & 1.93 & -0.93 & 0.54 & -5.33 & 4.81 & 231.87 & 0.55 & 0.48 & 0.31 & $728.56^{\star *}$ & $5233.86^{\star *}$ \\
\hline Misr 2 & 38.30 & 1.23 & -0.23 & 1.30 & -0.42 & $4.48^{\star *}$ & 198.30 & $1.58^{* \star}$ & 0.51 & 1.58 & 151.65 & $11290.42^{* *}$ \\
\hline Mean & 37.54 & & & & & & 226.16 & & & & & \\
\hline RLSD 5\% & 0.980 & & & & & & 11.54 & & & & & \\
\hline
\end{tabular}

Mean = grain mean yield (ardab/feddan); $\mathrm{bi}^{\mathrm{E}}=$ regression coefficient of Eberhart and Russell; $\beta_{\mathrm{i}}=$ regression coefficient of Perkins and Jinks; $\mathrm{b}_{\mathrm{i}}^{\mathrm{F}}=$ regression coefficient of Freeman and Perkins; $S^{2} d_{i}(E)=$ deviation from regression Eberhart and Russell; $S^{2} d_{i}(F)=$ residual MS of Freeman and Perkins model.*, ** Significant at the 0.05 and 0.01 probability levels, respectively. 

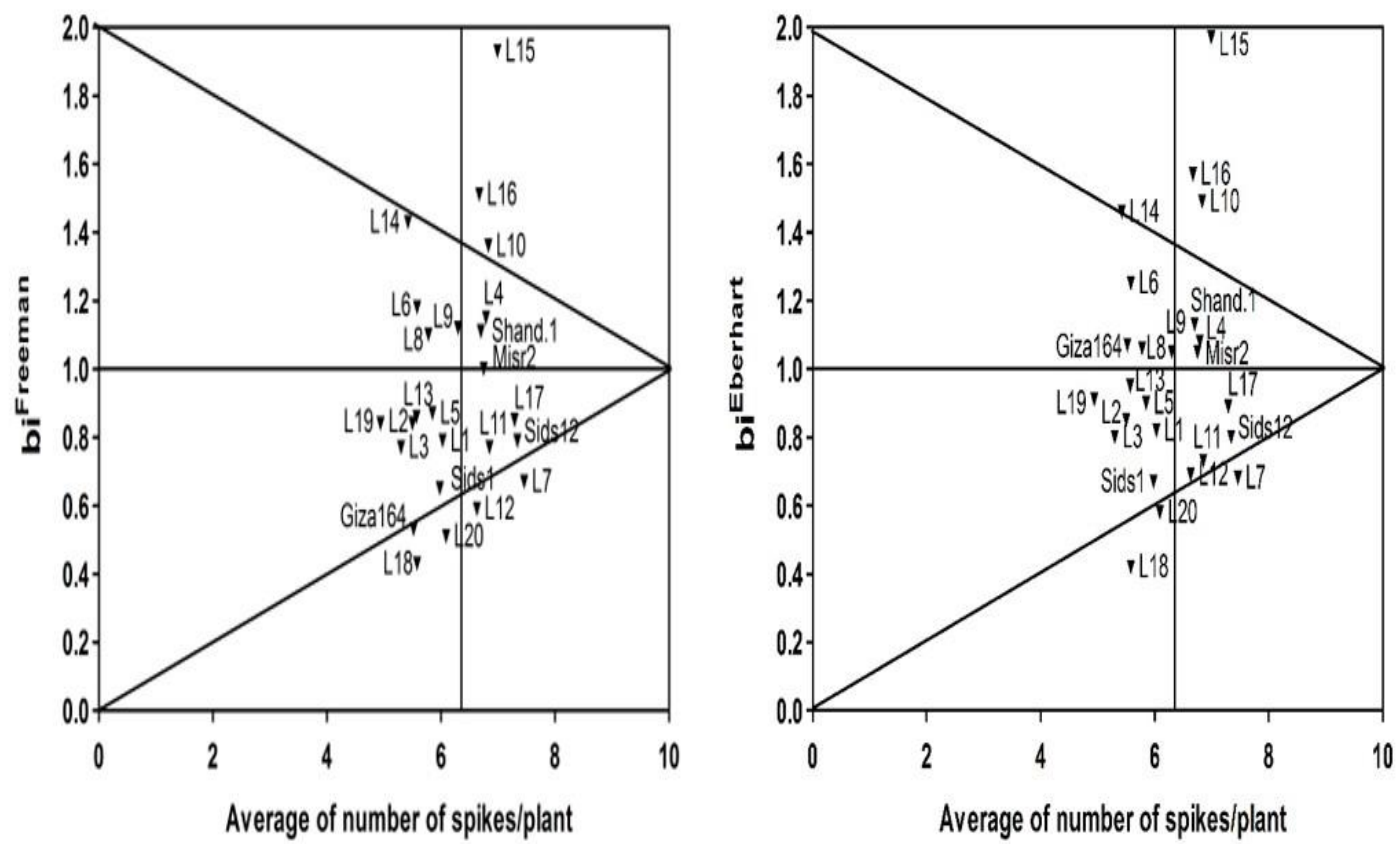

Figure1: Graphical illustration of the stability parameters $\left(\mathrm{bi}^{\text {Eberhart }}\right.$ and $\left.\mathrm{bi}^{\mathrm{Freeman}}\right)$ and the mean performance of individual genotypes for number of spikes/plant.
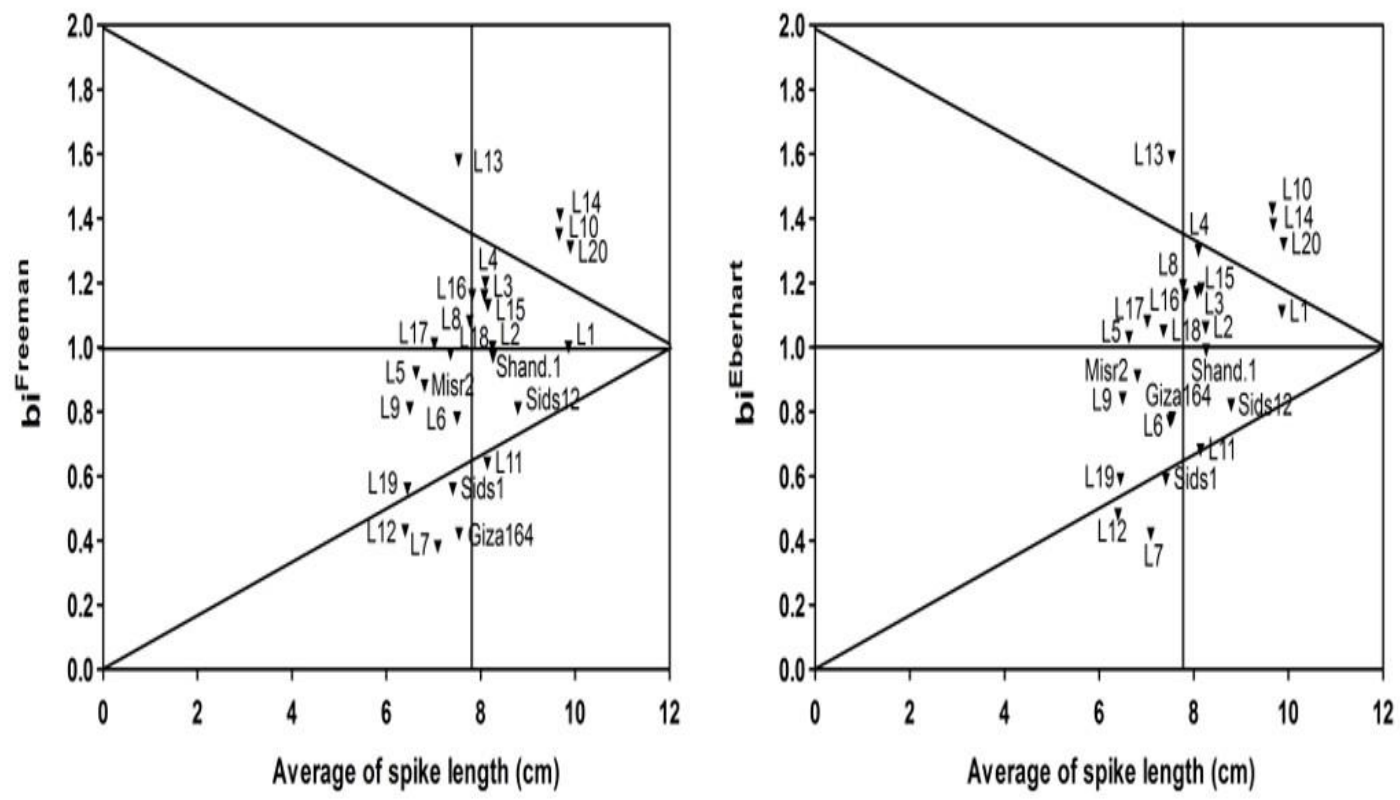

Figure 2: Graphical illustration of the stability parameters $\left(\mathrm{bi}^{\text {Eberhart }}\right.$ and $\left.\mathrm{bi}^{\text {Freeman }}\right)$ and the mean performance of individual genotypes for spike length.

namely Lines 2, 8, 9, 10, 11, 15, 17 and Shandweel 1 and Sids 1 (Table 4 and Fig. 3) exhibited stable performance due to their higher mean value than population mean and $\mathrm{b}_{\mathrm{i}}^{\mathrm{E}}$ equal or near to unity, Bi equal or near to zero and $\mathrm{S}^{2} \mathrm{di}^{\mathrm{E}}$ non-significant, reported by Eberhart and Russell's and Perkins and Jinks' models. According to slopes of independent linear regression coefficient (Freeman and Perkins, 1971), six genotypes namely Lines 2, 12, 13, 15, 16 and Shandweel 1 were stable over various environments because had biF equal or near to unity and $\mathrm{S}^{2} \mathrm{diF}$ non-significant with highest 1000 kernel weight when 
compared with grand mean. Saha, 1999 and Islam et al., 2006 found in pea got similar results for stability of 100 -seed weight in different genotypes using Eberhart \& Russell's and Perkins \&Jinks models. But Freeman \& Perkins model produced slightly different results.

\section{6- Chlorophyll concentration:}

The range of chlorophyll concentration varied from $33.23 \mathrm{mg} \mathrm{cm}^{-2}$ for Line 3 to $45.83 \mathrm{mg} \mathrm{cm}^{-}$

${ }^{2}$ for Line 20 with an average $40.42 \mathrm{mg} \mathrm{cm}^{-2}$ (Table 6). Five genotypes namely Lines 9,11, 16,17 and 18 were stable due to their $\mathrm{b}_{\mathrm{i}}^{\mathrm{E}}, \mathrm{Bi}$ and $\mathrm{S}^{2} \mathrm{di}^{\mathrm{E}}$ did not differ from a unit, zero and the zero, respectively plus significantly higher than mean over all genotypes (Table 4 and figure 4).Meanwhile, Lines 3, 8 and 10 were stable across various environments conditions but had mean lower than grand mean. According to Freeman and Perkins' model Line 9was stable performance, $b_{i}{ }^{F}$ equal or near to unity and $\mathrm{S}^{2} \mathrm{di}^{\mathrm{F}}$ nonsignificant with mean greater than overall mean chlorophyll concentration. While Line 11 also was stable, $\mathrm{b}_{\mathrm{i}}{ }^{\mathrm{F}}$ equal or near to unity and $\mathrm{S}^{2} \mathrm{~d}_{\mathrm{i}}{ }^{\mathrm{F}}$ nonsignificant but with mean lesser than the grand mean. Sharma et al., 2019 found five wheat genotypes showed stable performance not only for grain yield but also for associated characters like chlorophyll content.

\section{7- Specific leaf area:}

The average of specific leaf area across various environments (Table 4) was $226.25 \mathrm{~cm} \mathrm{gm}^{-}$ ${ }^{1}$ with a range from $198.24 \mathrm{~cm} \mathrm{~g}^{-1}$ for Line 10 to 247.24 for Line $14 \mathrm{~cm} \mathrm{gm}^{-1}$. According to Eberhart and Russell's and Perkins and Jinks' models (Table 4and Fig. 5), the genotypes Lines 3, 10 and Sids 12 exhibited stable performance because $b_{i}{ }^{\mathrm{E}}$ equal or near to unity, $B_{i}$ equal or near to zero, $S^{2} d_{i}^{E}$ non-significant and surpassed the grand mean of specific leaf area. Moreover, Line 20 and Shandweel 1 were specifically adapted to stress environmental conditions, regression coefficient lesser than unity $(b<1)$. While, Line 9 showed stability under favorable environment (surpassed the grand mean, $\mathrm{b}_{\mathrm{i}}^{\mathrm{E}}>1, \mathrm{~B}_{\mathrm{i}}>0$ and $\mathrm{S}^{2} \mathrm{~d}_{\mathrm{i}}^{\mathrm{E}}$ nonsignificant). While, according to Freeman and Perkins' model the genotypes namely Lines 2, 3, 10, 13 and Sids 12 surprised the grand mean and $b_{i}{ }^{F}$ equal or near to unity with significant deviations from regression coefficient $\left(\mathrm{S}^{2} \mathrm{~d}_{\mathrm{i}}^{\mathrm{F}}\right)$.

\section{8- Canopy temperature:}

The average canopy temperature of the different genotypes across various environments ranged from $24.28{ }^{\circ} \mathrm{C}$ for Line 17 to $29.50{ }^{\circ} \mathrm{C}$ for Line 15 with an average of $26.25^{\circ} \mathrm{C}$ (Table 5). According to Eberhart and Russell's and Perkins and Jinks' models, five genotypes namely Lines 3, 4, 8, Sids 12 and Giza 168 had colder plant canopy than grand mean over all genotypes beside $b_{i}{ }^{E}$ equal or near to unity, $B_{i}$ equal or near to zero, $S^{2} \mathrm{~d}_{i}{ }^{\mathrm{E}}$ non-significant. While, according to Freeman and Perkins' model the genotypes namely Line 3, Line 4, Sids 1 and Sids 12 surprised the grand mean and $b_{i}{ }^{\mathrm{F}}$ equal or near to unity but $\mathrm{S}^{2} \mathrm{~d}_{\mathrm{i}}^{\mathrm{F}}$ significant (Table 5and Fig. 6). Sharma et al., 2019 indicated that five wheat genotypes namely HD 2932, HI 1544, HD 2987 and Lok 1 were stable performance for leaf canopy temperature (had regression coefficient around unity $\left(b_{i}=1\right)$, non-significant deviation from regression $\left(S^{2} d_{i}\right)$, with high mean value than population mean, indicated its suitability and stability of performance under varied environments.

\section{9- Grain yield (ardab/feddan):}

The mean difference in grain yield (over all genotypes) between drought stress and nonstress are shown inTable6. Mean grain yield across varied environmental conditions ranged from 7.47 for Line 5 to 15.58 for Line 20 with an average of 11.77 ardab/feddan. According to two models viz., Eberhart and Russell's and Perkins and Jinks' (Table 6 and 
Table 5:.Estimates of stability parameters based on eight environments using various models for chlorophyll concentration and canopy temp. in wheat.

\begin{tabular}{|c|c|c|c|c|c|c|c|c|c|c|c|c|}
\hline Traits & \multicolumn{6}{|c|}{ Chlorophyll concentration $\left(\mathrm{mg} \mathrm{cm}^{-2}\right)$} & \multicolumn{6}{|c|}{ Canopy temperature $\left({ }^{\circ} \mathrm{C}\right)$} \\
\hline Genotypes & Mean & $\mathrm{b}_{\mathrm{i}}^{\mathrm{E}}$ & $\beta_{\mathrm{i}}$ & $\mathrm{b}_{\mathrm{i}}^{\mathrm{F}}$ & $\begin{array}{l}\mathrm{S}^{2} \mathrm{~d}_{\mathrm{i}} \\
(\mathrm{E})\end{array}$ & $\begin{array}{l}S^{2} d_{i} \\
(F)\end{array}$ & Mean & $b_{i}{ }^{\mathrm{E}}$ & $\beta_{\mathrm{i}}$ & $b_{i}{ }^{F}$ & $\begin{array}{l}\mathrm{S}^{2} \mathrm{~d}_{\mathrm{i}} \\
(\mathrm{E})\end{array}$ & $\begin{array}{l}S^{2} d_{i} \\
(F)\end{array}$ \\
\hline Line 1 & 36.58 & $0.44 * *$ & 0.56 & 0.27 & 0.74 & 24.33 & 25.94 & $1.41 * *$ & -0.41 & 1.23 & 0.60 & $9.54 * *$ \\
\hline Line 2 & 37.79 & $0.27 * *$ & 0.73 & $0.14 * *$ & 0.36 & $77.70 * *$ & 24.91 & $1.92 * *$ & -0.92 & $1.98 * *$ & 3.56 & $16.95 * *$ \\
\hline Line 3 & 33.23 & 0.96 & 0.04 & $0.83 *$ & 0.52 & $17.89 * *$ & 24.86 & 1.11 & -0.11 & 0.91 & 3.57 & $22.45^{* *}$ \\
\hline Line 4 & 35.21 & $1.40^{* *}$ & -0.40 & 1.45 & 0.75 & $14.40 * *$ & 24.86 & 1.14 & -0.14 & 0.92 & 1.69 & $17.54 * *$ \\
\hline Line 5 & 35.66 & $0.25 * *$ & 0.75 & 0.16 & 1.03 & 9.89 & 25.07 & $1.64 * *$ & -0.64 & 1.62 & 6.02 & $18.28 * *$ \\
\hline Line 6 & 35.43 & $0.24 * *$ & 0.76 & $0.57 *$ & 0.19 & 24.60 & 24.90 & 1.44 & -0.44 & 1.18 & $13.72 * *$ & $58.16^{* *}$ \\
\hline Line 7 & 35.69 & 1.39 & -0.39 & 1.25 & 0.80 & $21.08 *$ & 27.02 & $0.59 *$ & 0.41 & 0.35 & 4.01 & $28.06^{* * *}$ \\
\hline Line 8 & 37.38 & 0.86 & 0.14 & 0.54 & -0.71 & 2.77 & 26.24 & 1.03 & -0.03 & 0.83 & 2.27 & $18.26 * *$ \\
\hline Line 9 & 40.62 & 1.12 & -0.12 & 0.98 & -1.04 & 1.16 & 25.45 & $1.77 *$ & -0.77 & 1.52 & $6.98^{*}$ & $37.14 * *$ \\
\hline Line 10 & 36.91 & 1.11 & -0.11 & 0.94 & 0.75 & $19.99 * *$ & 25.35 & $1.37 * *$ & -0.37 & 1.38 & 1.26 & $12.51 * *$ \\
\hline Line 11 & 42.23 & 1.01 & -0.01 & 0.91 & 0.30 & 20.53 & 27.11 & $1.13 * *$ & -0.13 & 1.13 & -0.05 & $10.96 * *$ \\
\hline Line 12 & 42.10 & $1.62 * *$ & -0.62 & 1.42 & -0.08 & $3.72 * *$ & 27.75 & $0.35^{* *}$ & 0.65 & $0.49^{*}$ & -0.10 & 4.23 \\
\hline Line 13 & 44.57 & $0.80 * *$ & 0.20 & 0.76 & -0.22 & 1.46 & 27.94 & 0.85 & 0.15 & 1.12 & 1.43 & $11.80^{* * *}$ \\
\hline Line 14 & 41.51 & $0.63 * *$ & 0.37 & 0.59 & 0.48 & 9.54 & 28.13 & 1.36 & -0.36 & 1.64 & $8.01 *$ & $46.20 * *$ \\
\hline Line 15 & 44.18 & $1.19 *$ & -0.19 & $0.95^{* *}$ & 0.30 & $28.13 * *$ & 29.50 & 0.77 & 0.23 & 0.99 & $16.93 * *$ & $84.93 * *$ \\
\hline Line 16 & 43.09 & 1.13 & -0.13 & $1.29 *$ & 1.87 & $61.42 * *$ & 28.09 & 0.82 & 0.18 & 1.38 & $8.10^{*}$ & $40.68 * *$ \\
\hline Line 17 & 44.04 & 1.05 & -0.05 & 1.24 & -4.71 & $8.58 * *$ & 24.28 & 1.31 & -0.31 & 1.35 & 4.43 & $35.96 * *$ \\
\hline Line 18 & 42.83 & 1.01 & -0.01 & 1.06 & 0.03 & $9.06 * *$ & 25.46 & 0.72 & 0.28 & 0.63 & 3.28 & $25.77 * *$ \\
\hline Line 19 & 43.97 & $1.45^{* *}$ & -0.45 & 1.30 & 0.23 & $15.06 * *$ & 28.08 & 0.10 & 0.90 & 0.56 & $14.49 * *$ & $54.18 * *$ \\
\hline Line 20 & 45.83 & $1.53 * *$ & -0.53 & 1.43 & 2.08 & $33.60 * *$ & 28.12 & 0.44 & 0.56 & 0.74 & 6.25 & $31.15^{* *}$ \\
\hline Shandweel 1 & 44.37 & $1.32 * *$ & -0.32 & $1.37 * *$ & 0.33 & $48.89 * *$ & 24.37 & $0.69 *$ & 0.31 & 0.86 & -0.68 & $6.60 * *$ \\
\hline Sids 1 & 45.19 & 0.71 & 0.29 & 0.54 & 2.92 & 15.80 & 28.12 & 0.62 & 0.38 & 0.94 & 3.39 & $29.78 * *$ \\
\hline Sids 12 & 39.32 & 1.20 & -0.20 & 1.12 & 0.62 & $7.32 * *$ & 24.72 & 0.87 & 0.13 & 0.98 & -0.81 & $4.01 * *$ \\
\hline Giza 168 & 41.88 & $3.75 * *$ & -2.75 & $2.27 * *$ & -1.05 & -1.17 & 24.61 & 0.91 & 0.09 & 0.69 & 2.95 & $3.07 * *$ \\
\hline Misr 2 & 43.20 & $0.85^{*}$ & 0.15 & $0.60 *$ & 0.29 & $45.80 * *$ & 25.18 & $0.28 * *$ & 0.72 & 0.79 & 2.03 & $19.80 * *$ \\
\hline Mean & 40.52 & & & & & & 26.25 & & & & & \\
\hline RLSD 5\% & 1.14 & & & & & & 1.60 & & & & & \\
\hline
\end{tabular}

Mean $=$ grain mean yield $(\operatorname{ardab} /$ feddan $) ; b_{\mathrm{i}}^{\mathrm{E}}=$ regression coefficient of Eberhart and Russell; $\beta_{\mathrm{i}}=$ regression coefficient of Perkins and Jinks; $b_{i}{ }^{F}=$ regression coefficient of Freeman and Perkins; $S^{2} d_{i}(E)=$ deviation from regression Eberhart and Russell; $\mathrm{S}^{2} \mathrm{~d}_{\mathrm{i}}(\mathrm{F})=$ residual MS of Freeman and Perkins model.*, ** Significant at the 0.05 and 0.01 probability levels, respectively. 
Table 6: Estimates of stability parameters based on eight environments using various models for grain yield in wheat.

\begin{tabular}{|l|ll}
\hline Traits & Grain yield (ardeb/feddan)
\end{tabular}

\begin{tabular}{|c|c|c|c|c|c|c|c|c|c|c|c|c|c|c|}
\hline \multirow{3}{*}{$\begin{array}{l}\text { Traits } \\
\text { Genotypes }\end{array}$} & \multicolumn{14}{|c|}{ Grain yield (ardeb/feddan) } \\
\hline & \multicolumn{8}{|c|}{ Environments } & \multicolumn{6}{|c|}{ Stability parameters } \\
\hline & $E_{1}$ & $E_{2}$ & $E_{3}$ & $\mathrm{E}_{4}$ & $\mathrm{E}_{5}$ & $\mathrm{E}_{6}$ & $\mathrm{E}_{7}$ & $\mathrm{E}_{8}$ & Mean & $b_{i}^{E}$ & $\beta_{\mathrm{i}}$ & $b_{i}^{F}$ & $\mathrm{~S}^{2} \mathrm{~d}_{\mathrm{i}}(\mathrm{E})$ & $\mathrm{S}^{2} \mathrm{~d}_{\mathrm{i}}(\mathrm{F})$ \\
\hline Line 1 & 15.52 & 13.78 & 8.81 & 7.90 & 11.27 & 8.98 & 10.41 & 8.40 & 10.63 & $0.63^{\star \star}$ & -0.37 & 0.90 & -0.28 & 9.37 \\
\hline Line 2 & 16.69 & 15.76 & 12.66 & 12.61 & 12.79 & 12.28 & 9.17 & 7.43 & 12.42 & 0.71 & -0.29 & 0.74 & -0.12 & 7.88 \\
\hline Line 3 & 16.35 & 14.06 & 9.39 & 8.08 & 8.09 & 6.25 & 9.67 & 7.75 & 9.96 & 0.84 & -0.16 & 0.93 & -0.39 & 7.41 \\
\hline Line 4 & 15.21 & 11.21 & 10.05 & 8.55 & 9.83 & 7.75 & 6.69 & 6.05 & 9.42 & $0.69^{\star \star}$ & -0.31 & 0.72 & -0.46 & 5.46 \\
\hline Line 5 & 10.29 & 9.12 & 9.94 & 8.38 & 8.65 & 5.74 & 3.93 & 3.69 & 7.47 & $0.50^{* *}$ & -0.50 & $0.44^{*}$ & 1.60 & $14.74^{\star *}$ \\
\hline Line 6 & 12.80 & 11.97 & 8.39 & 5.93 & 9.22 & 8.73 & 8.49 & 7.18 & 9.09 & $0.52^{*}$ & -0.48 & $0.70^{*}$ & -0.81 & 7.86 \\
\hline Line 7 & 11.97 & 10.46 & 10.28 & 9.15 & 9.55 & 9.12 & 7.83 & 5.58 & 9.24 & $0.40^{\star \star}$ & -0.60 & $0.43^{*}$ & -0.79 & -0.52 \\
\hline Line 8 & 15.77 & 17.45 & 8.67 & 8.60 & 10.96 & 8.52 & 9.07 & 8.37 & 10.92 & 0.90 & -0.10 & 1.11 & -0.67 & 6.53 \\
\hline Line 9 & 17.54 & 21.91 & 11.48 & 13.66 & 11.29 & 9.86 & 10.62 & 9.30 & 13.21 & 1.08 & 0.08 & 1.12 & 0.51 & 11.18 \\
\hline Line 10 & 19.13 & 18.43 & 10.14 & 8.31 & 11.05 & 8.37 & 9.56 & 10.41 & 11.93 & 1.07 & 0.07 & 1.28 & 0.21 & $12.40^{*}$ \\
\hline Line 11 & 24.73 & 24.16 & 15.22 & 13.69 & 13.73 & 11.40 & 11.63 & 9.78 & 15.46 & $1.51^{* *}$ & 0.51 & 0.89 & -2.11 & 1.14 \\
\hline Line 12 & 12.49 & 17.09 & 8.96 & 10.61 & 8.07 & 9.74 & 8.70 & 7.82 & 10.44 & $0.70^{\star *}$ & -0.30 & 0.80 & 0.47 & 12.85 \\
\hline Line 13 & 21.81 & 21.90 & 10.00 & 9.10 & 10.81 & 10.37 & 9.47 & 9.83 & 12.91 & $1.41^{\star *}$ & 0.41 & 1.54 & 0.11 & $7.31^{\star *}$ \\
\hline Line 14 & 22.90 & 24.01 & 15.99 & 14.59 & 10.37 & 12.51 & 9.47 & 10.17 & 15.00 & $1.44^{\star *}$ & 0.44 & 1.89 & 0.22 & $9.73^{* *}$ \\
\hline Line 15 & 22.75 & 22.22 & 12.50 & 14.09 & 9.05 & 8.75 & 7.29 & 7.65 & 13.04 & $1.61^{\star \star}$ & 0.61 & 1.96 & -0.18 & $9.27^{\star \star}$ \\
\hline Line 16 & 18.09 & 13.29 & 8.44 & 7.00 & 8.37 & 8.20 & 9.86 & 8.86 & 10.26 & 0.87 & -0.17 & 1.00 & 1.70 & 17.46 \\
\hline Line 17 & 20.63 & 21.15 & 9.48 & 8.47 & 9.68 & 8.16 & 9.02 & 7.92 & 11.82 & $1.45^{\star *}$ & 0.45 & 1.63 & -0.69 & $5.77^{\star *}$ \\
\hline Line 18 & 11.51 & 10.73 & 8.92 & 8.31 & 9.73 & 9.81 & 7.80 & 6.40 & 9.15 & $0.36^{\star \star}$ & -0.64 & 0.42 & -1.35 & -3.94 \\
\hline Line 19 & 10.87 & 10.74 & 9.51 & 10.66 & 9.05 & 7.53 & 5.91 & 5.90 & 8.77 & $0.42^{\star \star}$ & -0.58 & $0.43^{\star \star}$ & -0.28 & 0.25 \\
\hline Line 20 & 24.58 & 23.96 & 14.64 & 14.54 & 12.98 & 12.86 & 10.53 & 10.11 & 15.58 & 1.48 & 0.48 & 1.74 & -2.09 & 1.66 \\
\hline Shandweel 1 & 23.68 & 23.77 & 14.35 & 14.30 & 11.01 & 11.58 & 10.38 & 9.91 & 14.87 & 1.48 & 0.48 & 1.25 & -1.57 & 3.75 \\
\hline Sids 1 & 19.11 & 19.37 & 13.29 & 11.80 & 11.34 & 11.32 & 9.78 & 9.53 & 13.19 & 1.02 & 0.02 & 1.10 & -2.13 & 1.67 \\
\hline Sids 12 & 23.76 & 21.70 & 12.82 & 12.50 & 10.55 & 10.66 & 10.01 & 9.64 & 13.95 & $1.45^{\star \star}$ & 0.45 & 1.22 & -1.75 & $2.15^{\star \star}$ \\
\hline Giza 168 & 20.95 & 19.75 & 13.18 & 13.23 & 10.24 & 10.33 & 9.24 & 9.20 & 13.49 & $1.91^{\star \star}$ & 0.91 & 0.96 & $-15.44^{*}$ & $2.63^{\star \star}$ \\
\hline Misr 2 & 17.64 & 17.22 & 12.21 & 11.80 & 10.14 & 9.89 & 8.03 & 8.35 & 11.91 & 0.96 & -0.04 & 1.03 & -1.82 & 2.97 \\
\hline mean & 17.87 & 17.41 & 11.17 & 10.63 & 10.31 & 9.55 & 8.90 & 8.21 & 11.77 & & & & & \\
\hline RLSD 5\% & 3.94 & 3.55 & 3.12 & 2.57 & 4.40 & 3.44 & 2.49 & 2.48 & 4.01 & & & & & \\
\hline
\end{tabular}

Mean = grain mean yield (ardab/feddan); $\mathrm{b}_{\mathrm{i}}^{\mathrm{E}}=$ regression coefficient of Eberhart and Russell; $\beta_{\mathrm{i}}=$ regression coefficient of Perkins and Jinks; $\mathrm{b}_{\mathrm{i}}^{\mathrm{F}}=$ regression coefficient of Freeman and Perkins; $S^{2} d_{i}(E)=$ deviation from regression Eberhart and Russell; $S^{2} d_{i}(F)=$ residual MS of Freeman and Perkins model.*, ** Significant at the 0.05 and 0.01 probability levels, respectively. 

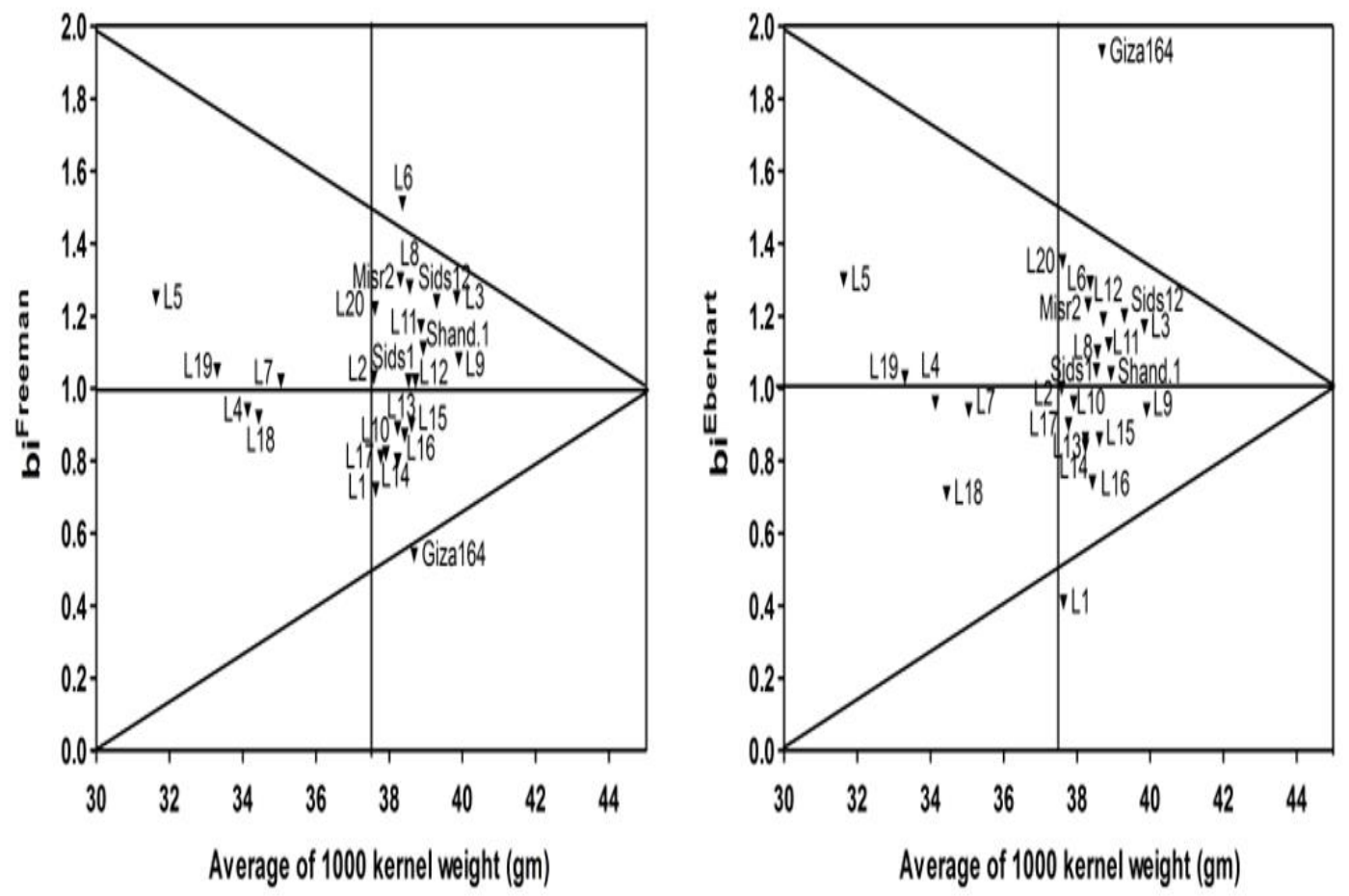

Figure 3: Graphical illustration of the stability parameters ( $\mathrm{bi}^{\text {Eberhart }}$ and $\mathrm{bi}^{\text {Freeman }}$ ) and the mean performance of individual genotypes for 1000 kernel weight.
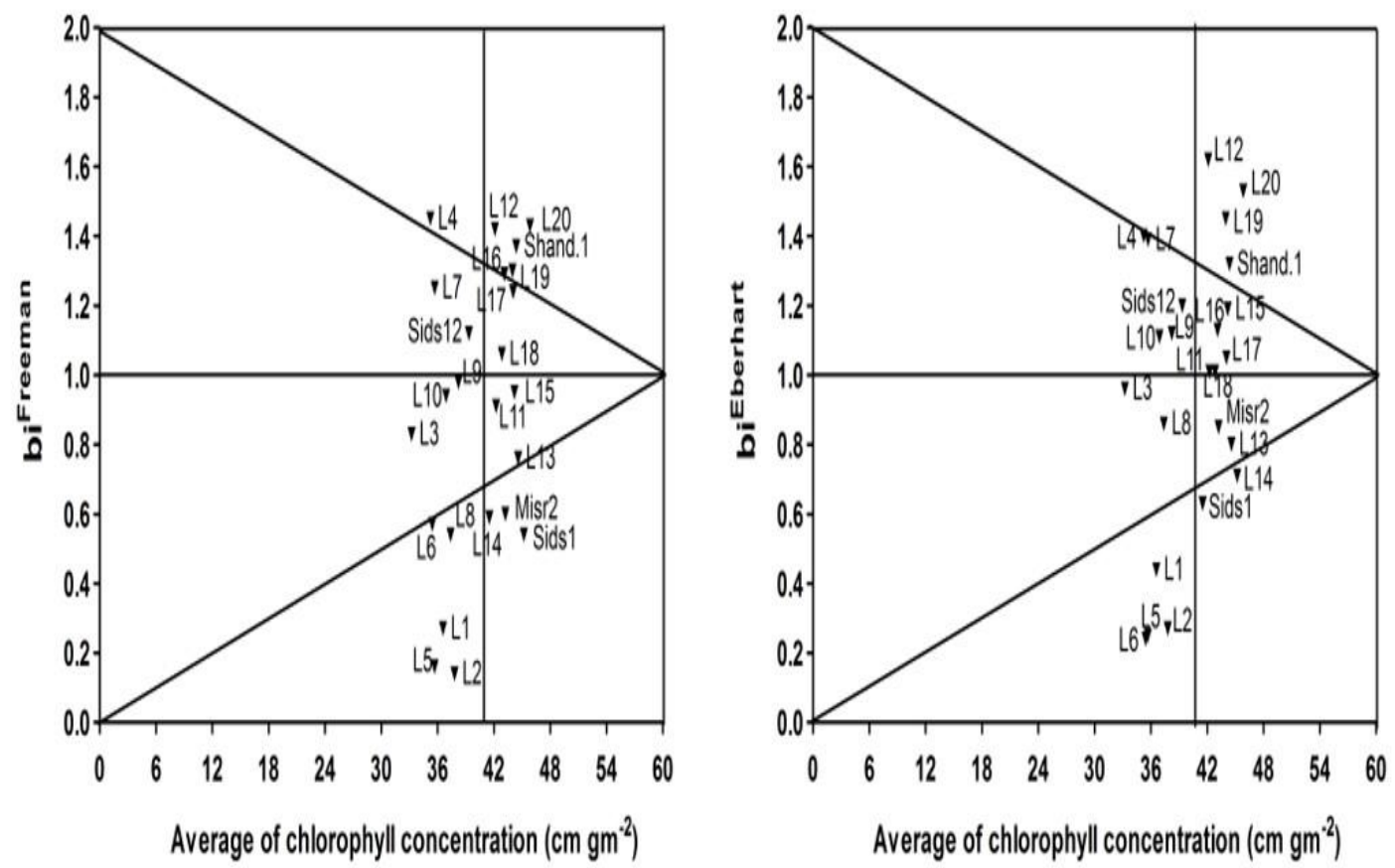

Figure 4. Graphical illustration of the stability parameters $\left(\mathrm{bi}^{\text {Eberhart }}\right.$ and $\mathrm{bi}^{\text {Freeman }}$ ) and the mean performance of individual genotypes for chlorophyll concentration. 

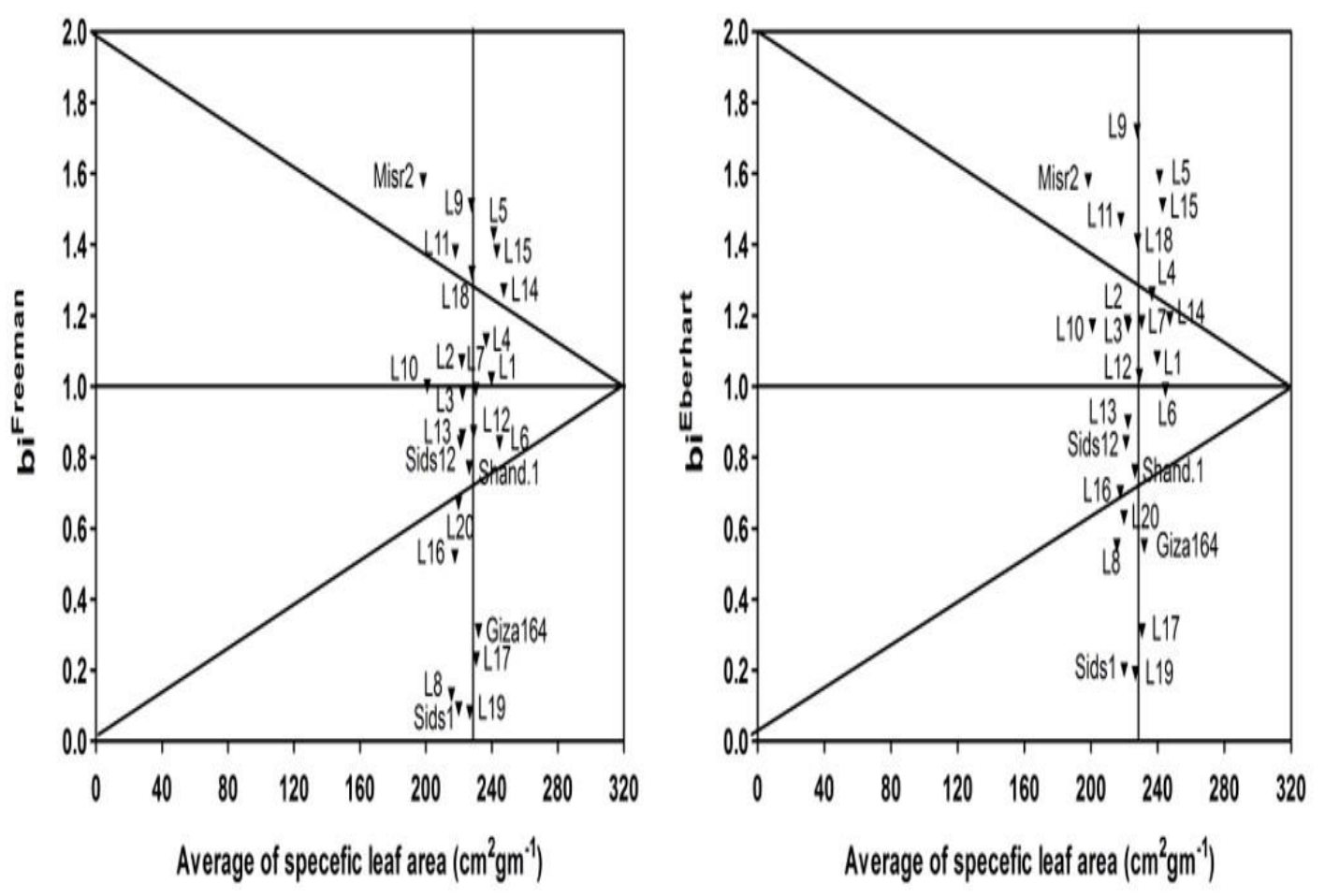

Figure 5: Graphical illustration of the stability parameters ( $\mathrm{bi}^{\text {Eberhart }}$ and $\mathrm{bi}^{\text {Freeman }}$ ) and the mean performance of individual genotypes for specific leaf area.
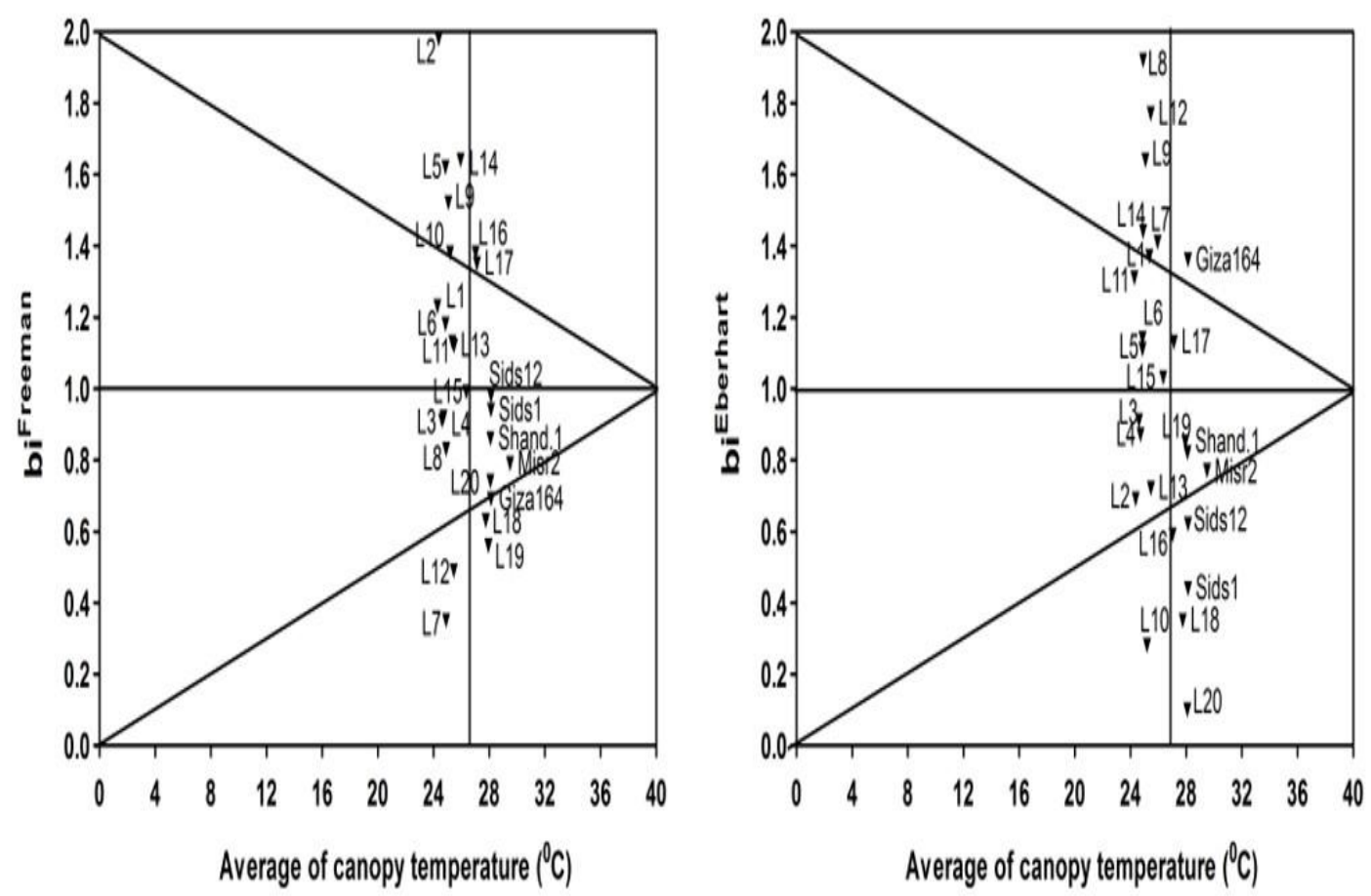

Figure 6: Graphical illustration of the stability parameters $\left(\mathrm{bi}^{\text {Eberhart }}\right.$ and $\mathrm{bi}^{\text {Freeman }}$ ) and the mean performance of individual genotypes for canopy temperature. 
Fig. 7), four genotypes namely Line 9, Line 10, Sids 1 and Misr 2 were stable over all the environments, whereas had grain yield above-grand mean, $b_{i}{ }^{\mathrm{E}}$ equal or near to one, Bi equal or near to zero and $\quad \mathrm{S}^{2} \mathrm{~d}_{\mathrm{i}} \mathrm{E}_{\text {non-significant. Four }}$ genotypes namely Line 9, Line 11, Sids 1 and Misr 2 showed also stable performance for grain yield across the environments, according to Freeman and Perkins' model (above grand mean, $\mathrm{bi}^{\mathrm{F}}$ equal or near to unity and $\mathrm{S}^{2} \mathrm{di}^{\mathrm{F}}$ nonsignificant). Moreover, two genotypes namely Line 20 and Shandweel 1 showed stability under favorable environment due to considering high mean performance with $\mathrm{bi}^{\mathrm{E}}$ and $\mathrm{bi}^{\mathrm{F}}>1$, $\mathrm{Bi}>0$ and $\mathrm{S}^{2} \mathrm{di}^{\mathrm{E}}$ and $\mathrm{S}^{2} \mathrm{di}^{\mathrm{F}}$ nonsignificant. While the genotype Line 2 exhibited stable performance under unfavorable environment (above grand mean, $b_{i}{ }^{\mathrm{E}}$ and $b_{i}{ }^{\mathrm{F}}<1, \mathrm{~B}_{\mathrm{i}}<0$ and $\mathrm{S}^{2} \mathrm{~d}_{\mathrm{i}}^{\mathrm{E}}$ and $\mathrm{S}^{2} \mathrm{~d}_{\mathrm{i}}^{\mathrm{F}}$ non-significant). Two genotypes (Lines 8 and 16) showed also stable performance over environments whereas, $b_{i}{ }^{\mathrm{E}}$ and $b_{i}{ }^{\mathrm{F}}$ near to $1, \mathrm{~B}_{i}$ near to 0 and $\mathrm{S}^{2} \mathrm{~d}_{\mathrm{i}}^{\mathrm{E}}$ and $\mathrm{S}^{2} \mathrm{~d}_{\mathrm{i}}^{\mathrm{F}}$ non-significant but with mean lowerthan the grand mean, according to three models. Pabale and Pandya (2010)indicated that the genotypes GHB-788, GHB-832 and GHB-840 were observed as most stable and widely adapted over environments in the models of Eberhart and Russell (1966), Perkins and Jinks (1968) and Freeman and Perkins (1971). Changizi et al. (2014) revealed that the selection of stable genotypes, based on these previous methods, caused high yield genotypes to be introduced as stable genotypes. These results are in agreement with those reported by Saha, (1999), Shinde et al. (2002), Chikurte et al. (2003), Yahaya et al. (2006), Islam et al., (2006) Mohammadi et al. (2012) and Karimzadeh et al. (2012).

\section{0- Correlation:}

The correlation (Table 6) indicated that the stability parameters, $b_{i}{ }^{\mathrm{E}}$ and $\beta i$ were similar $\left(\mathrm{r}=1.000^{* * *}\right)$ suggesting that both the methods (Eberhart\& Russell and Perkins \& Jinks) were identical. The ranking pattern for $b_{i}{ }^{F}$ values suggesting that Freeman and Perkins method was positive and highly significant with the previous two models $\left(\mathrm{r}=0.699^{* *}\right.$, Table $7)$. It can be seen from the Table 6 , that $\mathrm{b}_{\mathrm{i}}^{\mathrm{E}}\left(\mathrm{r}=0.855^{* *}\right), \beta_{\mathrm{i}}\left(\mathrm{r}=0.855^{* *}\right)$ and $\mathrm{b}_{\mathrm{i}}^{\mathrm{F}}$ $\left(\mathrm{r}=0.559^{* * *}\right)$ were positive and highly significantly associated with mean grain yield, while non-significant association of grain yield was observed with $\mathrm{S}^{2} \mathrm{~d}_{\mathrm{i}}^{\mathrm{E}}(\mathrm{r}=-0.299)$ and $\mathrm{S}^{2} \mathrm{~d}_{\mathrm{i}}^{\mathrm{F}}(\mathrm{r}=$ 0.175). It was also observed that $S^{2} d_{i}{ }^{E}$ was negative significantly associated with $\mathrm{bi}^{\mathrm{E}}\left(\mathrm{r}=-0.485^{* *}\right)$ and $\beta \mathrm{i}(\mathrm{r}=-$ $\left.0.485^{* *}\right)$ but non-significant with $\mathrm{b}_{\mathrm{i}}{ }^{\mathrm{F}}(\mathrm{r}$ $=0.0 .049$ ), while $\mathrm{S}^{2} \mathrm{~d}_{\mathrm{i}}^{\mathrm{F}}$ was nonsignificant with $\mathrm{bi}^{\mathrm{E}}, \beta \mathrm{i}$ and $\mathrm{b}_{\mathrm{i}}^{\mathrm{F}}$. Pabale and Pandya (2010) showed that the ranking patterns for $b_{i}{ }^{E}$ and $B_{i}$ were similar $(r=1.000)$ suggesting that both the methods (ER and PJ) were identical. The ranking pattern of genotypes using $\mathrm{b}_{\mathrm{i}}^{\mathrm{F}}$ values was also very close to the ranking pattern done under previous two models $(r=0.9655)$.

\section{Conclusion}

Our result emphasize that both mean performance of a genotype and its stability parameters should be taken together into consideration to identify 
Table 7: Correlation coefficients among various stability parameters for grain yield.

\begin{tabular}{|l|l|l|l|l|l|}
\hline Parameters & $\mathrm{b}_{\mathrm{i}}^{\mathrm{E}}$ & $\mathrm{S}^{2} \mathrm{~d}_{\mathrm{i}}^{\mathrm{E}}$ & $\beta_{\mathrm{i}}$ & $\mathrm{b}_{\mathrm{i}}^{\mathrm{F}}$ & $\mathrm{S}^{2} \mathrm{~d}_{\mathrm{i}}^{\mathrm{F}}$ \\
\hline Yield & $0.855^{* *}$ & -0.299 & $0.855^{* *}$ & $0.559^{* *}$ & -0.175 \\
\hline $\mathrm{b}_{\mathrm{i}}^{\mathrm{E}}$ & - & $-0.485^{* *}$ & $1.000^{* *}$ & $0.699 * *$ & -0.055 \\
\hline $\mathrm{S}^{2} \mathrm{~d}_{\mathrm{i}}^{\mathrm{E}}$ & - & - & $-0.485^{* *}$ & 0.049 & $0.413^{*}$ \\
\hline$\beta_{\mathrm{i}}$ & - & - & - & $0.699 * *$ & -0.056 \\
\hline $\mathrm{b}_{\mathrm{i}}^{\mathrm{F}}$ & - & - & - & - & 0.277 \\
\hline
\end{tabular}

$*$,** Significant at the 0.05 and 0.01 probability levels, respectively.
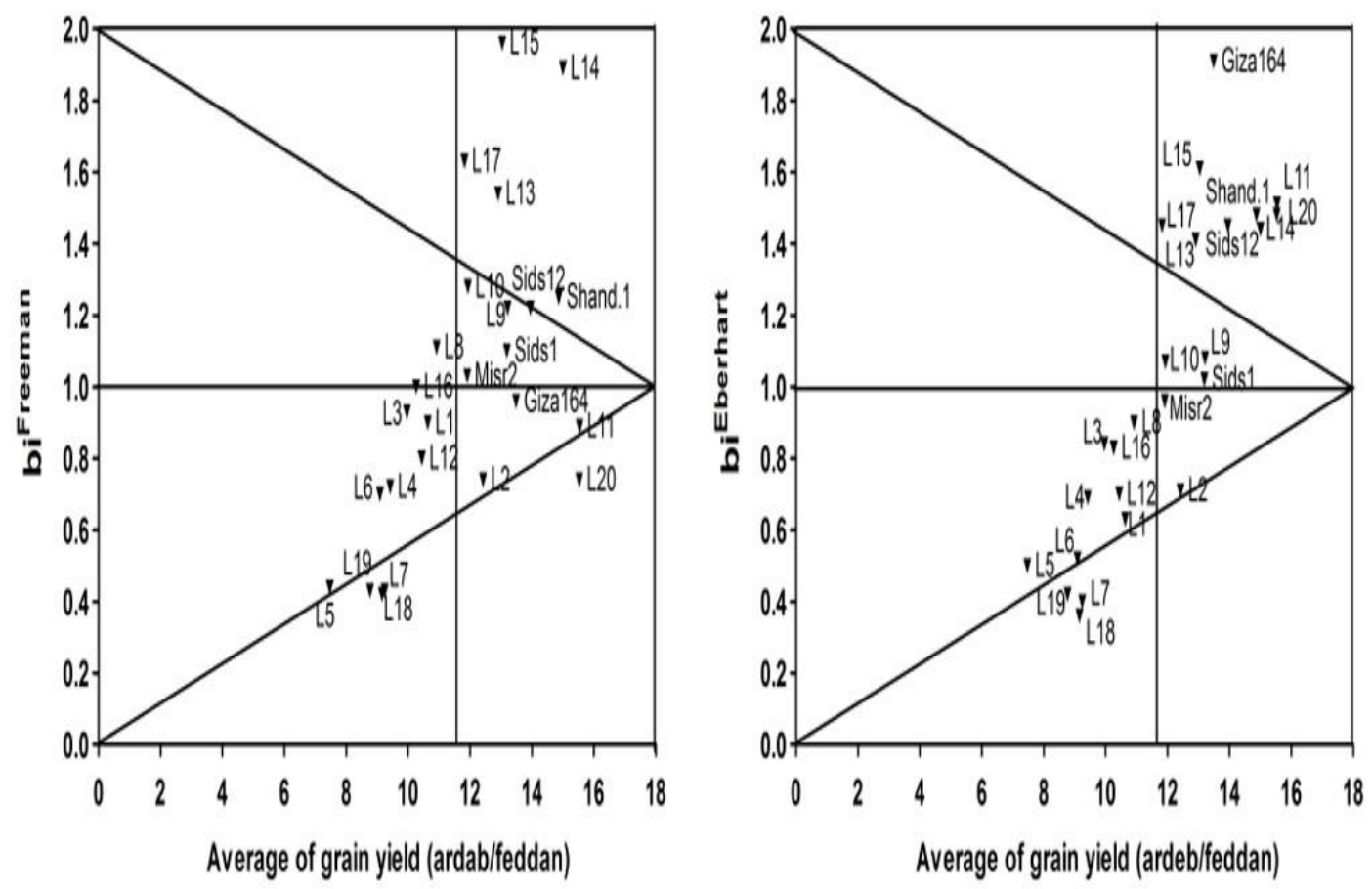

Figure 7: Graphical illustration of the stability parameters (bi ${ }^{\text {Eberhart }}$ and bi $^{\text {Freeman }}$ ) and the mean performance of individual genotypes for grain yield.

new genotype to be used in various environments. Whereas, according to three models viz., Eberhart and Russell's, Perkins and Jinks' and Freeman and Perkins', Line 9 (13.21 ardab/feddan) beside Sids 1 (13.19 ardab/feddan) and Misr 2(11.91 ardab/feddan) cultivars were observed as most stable and widely adapted over environments, surprised mean performance for grain yield than population mean, $b_{i}{ }^{\mathrm{E}}$ and $b_{i}{ }^{\mathrm{F}}$ equal or near to one, $\mathrm{B}_{\mathrm{i}}$ equal or near to zero and $\mathrm{S}^{2} \mathrm{~d}_{\mathrm{i}}^{\mathrm{E}} \quad$ and $\quad \mathrm{S}^{2} \mathrm{~d}_{\mathrm{i}}^{\mathrm{F}}$ non-significant. Moreover, Line 9 showed also stability for number of spikes/plant, 1000 kernel weight and chlorophyll concentration under different environments, according to three models. So, it was considered to be superior for grain yield across various environments. Meanwhile, the highest yielding genotype (Line 20) only was suitable for favorable environments.

\section{References}

Alwala, S., Kwolek, T., McPherson, M., Pellow, J., and Meyer, D. (2010)' A comprehensive comparison between Eberhart and Russel joint regression and GGE biplot analyses 
to identify stable and high yielding maize hybrids', Field Crop. Res., (119), pp. 225-230.

Bose, L.K., Jambhulkar, N. N., Pande, K. and Singh, O. N. (2014)'Use of AMMI and other stability statistics in the simultaneous selection of rice genotypes for yield and stability under direct-seeded conditions', Chilean J. Agric. Res. 74 (1) Chillán mar., online ISSN 0718-5839.

Changizi M., Choukan, R., Heravan,E. M.,Bihamta,M. R. and Darvish, F. (2014) 'Evaluation of genotype $\mathrm{x}$ environment interaction and stability of corn hybrids and relationship among univariate parametric methods',Can. J. Plant Sci., (94), pp. 1255-1267.

Chaves, M., Maroco, J.P., Pereira, J.S. (2003) 'Understanding plant responses to drought - from gene to whole plant', Functional Plant Biology(30), pp. 239-264. doi:10.1071/FP02076.

Chikurte, K. N., Desale, J. S. and Anarase, S. A. (2003)'Genotypic x environment interaction for yield and yield components in pearl millet', J. of Maharashtra Agric. Uni., 28(1), pp. 30-33.

Condon, A.G., Richards, R.A., Rebetzke, G.J., Farquhar, G.D. (2004) 'Breeding for high water use efficiency', Journal of Experimental Botany (55), pp. 2447-2460. doi:10.1093/jxb/erh277.

Dehghani, H., Sabaghpour, S. H. and Sabaghnia, N. (2008) 'Genotypeenvironment interaction for grain yield of somelentil genotypes and relationship among univariatestabilitystatistics', Span. J. Agric. Res., (6), pp. 385-394.

Eberhart, S. A. and Russell, W. A. (1966) 'Stability parameters for comparing varieties', Crop Sci., (6), pp. 36-40.

EI-Morshidy, M.A., Elorong, E.E.M., Tammam, A.M. and Abd EI-Gawad, Y.G. (2000) 'Analysis of genotype X environment interaction and assessment of stability parameters of grain yield and its components of some wheat genotypes (Triticum aestivum L.) under new valley conditions', The 2nd Scientific Conf. of Agri. Sci., Oct., Assiut, pp. 13-34.
FAO STAT (2018) 'Food and Agriculture Organization of the United Nations (FAO), FAO Statistic Database', http://www.fao.org/faostat.

Fisher, R.A. and Yates, F. (1953) 'Statistical Tables for Biological, Agricultural, and Medical Research'," Olive and Boyd", Edinbugh, pp.94.

Freeman, G. H. and Perkins, J. M. (1971) 'Environmental and genotype-environmental components of variability VIII. Relations between genotypes grown in different environments and measures of these environments', Heredity (27), pp.15-23.

Gomez, K. A. and Gomez, A. A. (1984) 'Statistical Procedures for Agricultural Research', 2nd Ed., John Wiley and Sons, New York, USA.

Ibrahim, Kh.A.M. and Said, A.A. (2020) 'Grain Yield Stability of New Bread Wheat Genotypes (Triticumaestivum L.) under Normal and Heat Stress Conditions', Egypt. J. Agron., 42(2), pp. 171-184.

Islam,A. K. M. A.,Newaz,M. A.,Jahan, M. M. A. and Islam, M. A. (2006)'stability analysis of seed yield in dry bean (phaseolus vulgaris 1.) genotypes following different models', Bangladesh J. Pl. Breed. Genet., 19(1), pp. 07-14.

Karimizadeh, R., Mohammadi, M., Sabaghnia, N., Shefazadeh, M. K. and Pouralhosaini, J. (2012)'Univariate stability analysis methods for determining genotype $\mathrm{x}$ environment interaction of durum wheat grain yield', Afr. J. Biotechnol., (11), pp. 2563-2573.

Khan, F., Khan, M. I., Khan, S., Zaman, M. A., Rasheed , H., Khan, A. (2017) 'Evaluation of agronomic traits for yield and yield components in wheat genotypes with respect to planting dates', Malaysian J. of Sustainable Agri., 2(1), pp. 07-11.

Kheiralla, K.A., EI-Morshidy, M.A., Motawea, M.H. and Saeid, A.A. (2004) 'Performance and stability of some wheat genotypes under normal and water stress conditions', Assiut J. Agric. Sci., 35 (2), pp. 74- 94. 
Moghaddam, A., Raza, A., Vollmann, J., Ardakani, M. R.,Wanek, W., Gollner, G. and Friedel, J. K. (2013) 'Carbon isotopediscrimination and water use efficiency relationships of alfalfa genotypes under irrigated and rain-fed organic farming', Eur. J. Agron., (50), pp. 82-89.

Mohamed, N.E. M. and Said A. A. (2014) 'Stability Parameters for Comparing Bread Wheat Genotypes under Combined Heat and Drought Stress',Egypt. J. Agron., 36 (2), pp. $123-146$.

Mohammadi, M., Karimzadeh, R., Sabaghnia, N. and Shefazadeh, M. K. (2012)'Genotype x environment interaction and yield stability analysis of new improved bread wheat genotypes', Turk. J. Field Crops. (17) pp. 67-73.

Ongom, P. O., Volenec, J. J. and Ejeta, G. (2016)'Selection for Drought Tolerance in Sorghum Using Desiccants to Simulate PostAnthesis Drought Stress', Field Crops Res., 198, pp. 213-321.

Pabale, S. S. and Pandya, H. R. (2010) 'A comparison of different stability models for genotype $\mathrm{x}$ environment interaction in pearl millet', Electronic Journal of Plant Breeding, 1(5), pp. 1294-1298.

Perkins, J. M. and Jinks, J. L. (1968) 'Environmental andgenotypeenvironmental components of variability', Heredity (23), pp. 339356.

Richards, R.A. (2006) 'Physiological traits used in the breeding of new cultivars for water-scarce environments', Agricultural Water Management, (80), pp. 197-211. doi:10.1016/j.agwat.2005.07.013.

Saha, G. C. (1999)'Genotypeenvironment interaction and in vitro regeneration in pea', M. S. Thesis. Dept. Genetics and Plant Breeding, BAU, Mymensingh.,pp.75.

Sharma, V., Dubey, R. B. and Khan, R. (2019) 'Genotype-environment interaction on stability of grain yield and physio-biochemical traits in bread wheat (Triticumaestivum L.)', Bangladesh J. Bot., 48(4), pp. 11431151 .

Shinde, G. C., Bhingarde, M. T., Khairnar, M. N. and Mehetre, S. S.
(2002)'AMMI analysis for stability of grain yield of pearl millet hybrids', Indian J. Genet., 62(3), pp. 215-217.

Steel, R. G. D., and Torrie, J. H. (1980) 'Principles and Procedures of Statistics.McGraw Hill Book Company', Inc. New York.

USDA STAT (2018) 'USDA National Agricultural Statistics Service Information', https://www.nass.usda.gov/Publicati ons/Ag Statistics/2018/index. Php.

Werteker, M., Lorenz, A., Johannes, H., Berghofer, E. and Findlay, C. S. (2010)'Environmental and Varietal Influences on the Fatty Acid Composition of Rapeseed, Soybean and Sunflowers', J. Agron. Crop Sci., (196), pp. 20-27.

Yahaya, Y., Echekwu C. A. and Mohammed, S. G. (2006)'Yield stability analysis of pearl millet hybrids in Nigeria', African $J$. Biotech., 5(3), pp. 249-253. 